\title{
Application of Ant Colony Algorithm in Fingerprint Identification System Based on Internet of Things
}

\author{
Zhigao Chen \\ Hu Nan occasional institute of science \& technology, \\ Changsha, China, 410018 \\ Chenzhigao7621414@163.com
}

\begin{abstract}
The Internet of things is a network which connected to objects through the Internet, internet was treated as the foundation and the core ,by which the most date transmission is wireless information and intelligent processing information, in this situation for one thing, the social efficiency can greatly improved, for another, the problem of security such as identity authentication and privacy issues. Fingerprint identification can be effectively applied in identity authentication, while there is a high rate of false rejection and false acceptance in traditional fingerprint identification method. An adaptive ant colony algorithm was present in this paper, by which the threshold was set. The image segmentation fingerprint sample image was devide to blocks in the client, and were sent to the server for matching. As the experimental results shown, the system can effectively improve the recognition rate of fingerprint remotely.
\end{abstract}

Keywords-component;internet of Things; Ant colony algorithm; Pheromone;

\section{INTRODUCTION}

The Internet of things is accomplished by sensing device information, according to the agreed protocol implementation person and person, person and matter, and full interconnection network, which is mainly characterized by RFID, sensor such as access to the physical world of information, combined with

Internet, mobile communication networks and other networks for the transmission of information and interaction, intelligent computing technology to analyze and process the information, thereby improving the material world perception ability, realize the intelligent decision and control. Since 2009 August Premier Wen Jiabao proposed" the perception of China" since, the Internet of things was officially listed

as the national five large emerging strategic industries, writes the" government work report", the Internet of things in China by the whole society greatly Attention, 2010 during two meetings, once again become a hot topic of Internet of things. As the perception of Chinese strategy of start-up and gradual development, networking market now from power, transportation, security and other public services gradually into the civilian market of various industries, Chinese Internet industry development faces great opportunities.

\section{ANT ALGORITHM INTRODUCED}

Ant algorithm is by the Italian scientist M. Dori ants find food by nature, in the experience left on the path of information has always been called Fireman call other ants to reach the food source incident inspired the formation of a positive feedback optimization algorithm. This method has been widely used in optimal path in optimal

path searching, NP problem solving, and image segmentation study. This paper presents adaptive algorithm for the search area ant block, each block image image smoothing, enhancing and refining pre-treatment process is to set a threshold, For the matching success rate is greater than the threshold that the authentication is successful, otherwise the task of matching failure. The algorithm can effectively reduce the traditional image of the fingerprint. identification process rejection rate and error rate

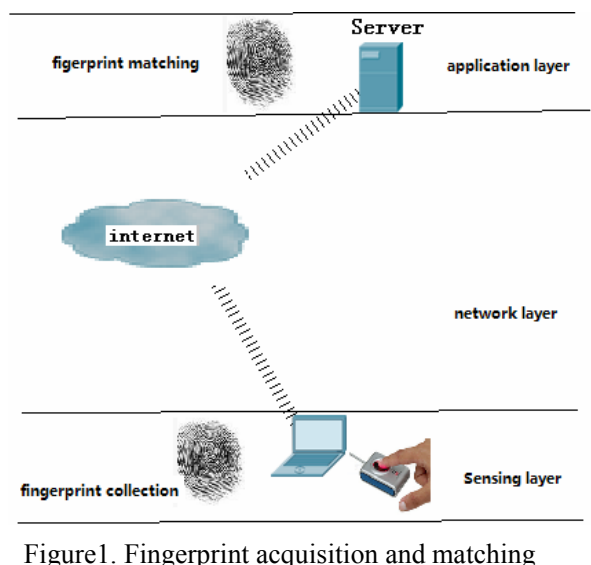

\section{THE PRINCIPLE OF FINGERPRINT IDENTIFICATION SYSTEM}

\section{A. Fingerprint Identification System structure}

As Shown in Figure 1, this paper designed by fingerprint identification system is configured on the client laptop fingerprint scanner, and then collected by the ant algorithm for fingerprint samples are processed through the wireless network to the server, and server-side standard pre-stored fingerprint samples matched, if the match is successful the information to confirm the identity of the sender, if unsuccessful, after been to match the sender can confirm the illegal users.

\section{B. Adaptive image segmentation algorithm ant ideas}

Traditional ant algorithm for image matching, generally requires the whole image all pixels are shown in Figure 2 requires a $3 \times 3$ neighborhood scanning, As a scan of the pixels are too many, the image segmentation process is very 
slow. In this paper, adaptive ant algorithm for image segmentation is not necessary to the whole image is scanned once and for all, but the image is divided into several blocks, each of which will scan an area after the split directly to the server and the database has been exist to match the standard fingerprint samples. By the graph theory of knowledge can know exactly if the two plans overlap, then one must also figure as part of a picture with a part of another complete coincidence, on the contrary, if a picture in another part of a whole map can not find an exact match on the part of the two maps is certainly not an exact match. Therefore, if the sample was collected pre-stored in the database can be found in the sample is bound to match the standard sample (if not found then the collection of samples and standard samples do not match), then the rest of the block can be sent to the server to continue match, matching success ultimately depends on whether the pre-set threshold, such as setting the threshold value of greater than $80 \%$ is considered an exact match, then for an image is divided into nine of which eight matches as long as samples can be collected into that match with the standard sample.

\section{A Self-Adaptive Clustering Algorithm Based on Ant Swarm Image Segmentation Process}

For the collected in accordance with the fingerprint image can be divided into 3 lines of 3 columns, mean nine lump,In the nine blocks as take of a cake.

In the choice of the rectangular the image. set some piexl point,each piexl point The search area can use the following plan:set datum mark piexl is Xij,eEach area of the ant search for $(I-t, I+t)$ width and $(j-k, j+k)$ length of the area and then 8 direction search of as shown in figure 2,calculate the minimum gradient value Tmin image,then calculate the between gradient of pixels and Tmin amplitude value difference.

Will be smaller than these D-value of the threshold value " $\mathrm{H}$ " as the "food" from the ants to in search. When the ant for to seek after the goal, that is found the edge pixels. as for "di" set

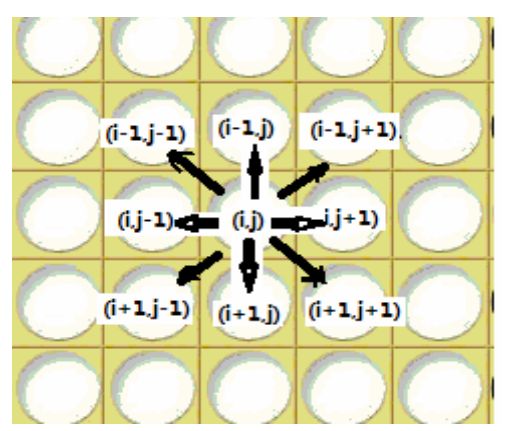

Figure2. Image segmentation

gradient of pixel to minimum gradient value Tmin distance. exactly:

$$
d_{i}=\left|\mathrm{T}_{i}-\mathrm{T}_{\min }\right|
$$

In traditional ant algorithm, Fairmon have stay in the ant search route, this algorithm has been modified, will be in by the ants Fairmon each pixel. " $\mathrm{i} i$ " for information, $\mathrm{H}$ for threshold value, exactly:

$$
\tau_{i}= \begin{cases}1 & d_{i} \leq \mathrm{H} \\ 0 & d_{i}>\mathrm{H}\end{cases}
$$

For di $>\mathrm{H}, \quad X_{i j}$ referred to as target internal or background area (the edge area) probability is

$$
p_{i}= \begin{cases}\frac{\tau_{i}^{a}(t) \eta_{i}{ }^{\rho}(t)}{\sum_{i e s} \tau_{i}^{a}(t) \eta_{i}{ }^{\rho}(t)} & i \in S \\ 0 & \text { otherwise }\end{cases}
$$

$\eta \mathrm{I}(\mathrm{t})$ of the calculation method is:

$$
\eta_{i}(t)=\frac{\mathrm{H}}{d_{i}}
$$

Thereinto, $\mathrm{H}$ is gradient threshold value, $\eta$ and $\mathrm{H}$ is directly proportional, and to the di is inversely proportional. also $\alpha$ and $\beta$ for two parameters, the pixels are respectively placed, be classified as a edge in the process of the accumulated by the information. $S=\{X i j \mid d i \leq H, i=1,2, \cdots, m$ $\times \mathrm{n}$ \} for optional collection of pixels. With the movement of the ants, each pixel information on the change. Every complete a cycle, each pixel is the Fairmon have according to the type to adjust:

$$
\begin{aligned}
& \tau_{i}(t+1)=\rho \cdot \tau_{j}(t)+\Delta \tau_{i} \\
& \Delta \tau_{i}=\sum_{i=1}^{m \times n} \Delta \tau_{i}^{k}
\end{aligned}
$$

Thereinto, (1- $\rho)$ for a pheromone's attenuation coefficients, usually set $\rho<1$ to avoid pixels on the infinite increase with Fairmon, $\Delta \tau_{i j}$ means the cycle of pixels with the increment of the Fairmon, $\Delta \tau^{k}{ }_{i}$ means the first $\mathrm{k}$ ant in this cycle in the pixel when Fairmon have.

The ant search path with the increase of cycling times to segmentation image edge gradually convergence.the ant colony algorithm for target path in performance from the pheromone significantly greater than other areas,therefore according to the element information distribution extraction separation results.As pecified in the iteration number after and cycling times, if the pheromone of the node (I, j) meet: $\tau$ $i, j^{\prime \prime} \geq \tau$ max . The node (I, j) mark for the target, or as the background.

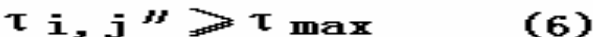

\section{D. algorithmic process}

A (1)initialization, the number $m$ of ants ant random distribution of pixels in the image on the cycles of $\mathrm{K}$, the number of iterations $\mathrm{N}$, the initial moments of the path of the pheromone to $\tau(0) \mathrm{i}, \mathrm{j}=\tau \max$; initialization parameters $\alpha, \beta, \rho, \mathrm{H}, \mathrm{R}$.

B (2)fingerprint samples to be processed into gray-scale images, for its smooth, enhance, refine, etc. treatment, the results shown in Figure 3 the results of the samples change plans.

C (3)According to (1) calculated pixel Xij of di. If di is 0 , then the pixels are classified as non-edge area of the membership degree of 1 ; if $\mathrm{di} \leq \mathrm{H}$, then according to equation (3) calculate the pixel Xij be classified as target or background within the probability area, which will have the greatest probability classified as background pixels Xij area. 
D (4)for $\mathrm{di}>\mathrm{H}$, then setting a grayscale threshold $\mathrm{R}$, is used to distinguish the noise pixel is a border point or points.

E(5)According to equation (4) and (5) calculation Fireman incremental update Fireman volume.

$\mathrm{F}(6)$ to determine whether there are not classified as a pixel, if the loop, until all pixel coordinates traverse the entire region.

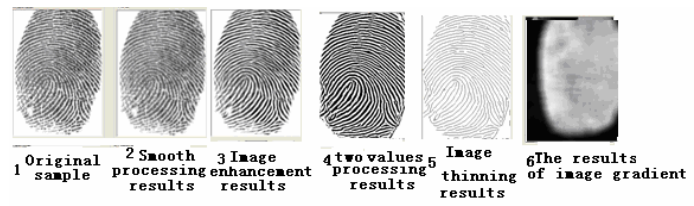

Figure3. A variety of fingerprint processed results

G (7)According to formula (6) the image processed by image segmentation.

Based on Figure 1 in the network topology, using Visual $\mathrm{C}++6.0$ image acquisition written client program and server-side program, and pre-stored on the server 20 in the standard image of the fingerprint sample into the sample library, each sample number, parameter is set to $\alpha=1, \beta=1$, $\mathrm{C}=1, \rho=0.05$, matching threshold is set to $85 \%$ segmentation block number 9 , the first client device through fingerprint fingerprint samples sent to the server side, the run shown in Figure 4, the server receiving the transmission over after a fingerprint sample and the sample library to match the standard sample, if the match criteria for a successful match is displayed in the sample number, the operating results shown in

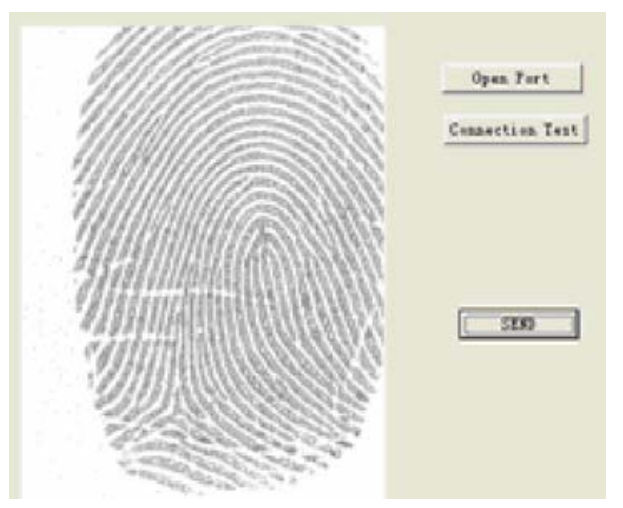

Figure4. Fingerprint identification procedure

Figure 5. Through the sample library to match the 20 samples, more than $90 \%$ efficiency.

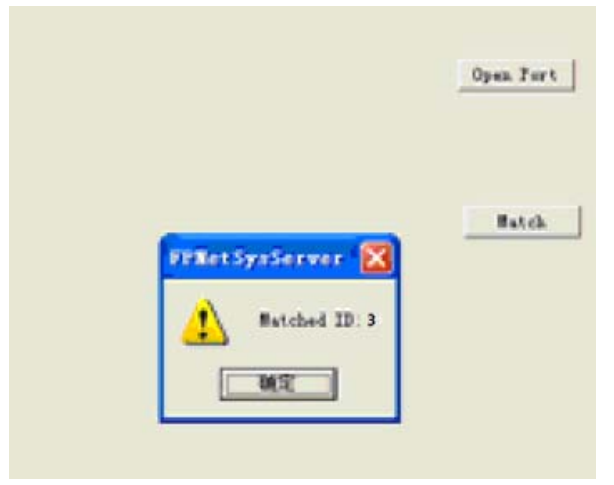

Figure5. Fingerprint identification result

\section{Conclusion}

Through the above experiments show that using adaptive ant algorithm for fingerprint recognition fingerprint identification system is better, the system can be applied to the remote user's fingerprint, the user only needs a laptop computer coupled with fingerprint and other equipment can be as in the field as effective as fingerprint identification. As the fingerprint image acquisition time during the inevitably

because of the hands sweat collectors and other external disturbances on the recognition rate to a certain extent, these factors are to

study this in the future and improvement. Another set thresholds and parameters will also affect the recognition success rate, this threshold is not optimal to do a deeper study, which is the follow-up to complement this work..

\section{References}

[1] Finkenzeller K.RFID Handbook.New York:Wiley,1999.

[2] Nezamabadi-pour H,Saryazdi S,and Rashedi E.Edge detection using ant algorithm [J].Soft Comput,2006,10(7):623-628

[3] DORIGO M,GAMBARDELLA L M. Ant colony sys-tem:a coorperative learning approach to thetraveling salesman problem $[\mathrm{J}]$.IEEE Trans onEvolutionary Com-putation, 1997,1(1):53-66.

[4] M.Satyanarayanan,Pervasive Computing:Vision and Challenges[J] ,IEEE PersonalCommunications, August 2001:10-17.

[5] Nigel Shadbolt,Ambient Intelligence[J],IEEE INTELLIGENT SYSTEMS, JULY/AUGUST.2003:1-3.

[6] Meshoul S and Batouche M.Ant colony system with extremal dynamics for point matching andpose estimation.Proceedings of the $16^{\text {th }}$ International Conference on Pattern Recognition,Los Alamitos,USA,2002,3:823-826. 